\title{
Non-emptiness of the alpha-core: sufficient and necessary conditions
}

\author{
Achille Basile $^{1} \cdot$ Vincenzo Scalzo $^{1}$
}

Accepted: 1 September 2020 / Published online: 18 September 2020

(c) The Author(s) 2020

\begin{abstract}
We give sufficient and necessary conditions for the non-emptiness of the alpha-core in the setting of strategic games with non-ordered and discontinuous preferences. In order to prove our results, we can avoid the use of Scarf's Theorem for NTU-games, by suitably appealing to the Ky Fan minimax inequality. Examples clarify our conditions and allow the comparison of our results with the previous ones.
\end{abstract}

Keywords Games with non-ordered preferences · Discontinuous games ·

Non-emptiness of the alpha-core

JEL Classification C60 - C71

\section{Introduction}

A well known cooperative solution concept for strategic form games is the $\alpha$-core by Aumann (1961). A strategy profile belongs to the $\alpha$-core if no coalitions of players have profitable joint deviations against to all strategies of the opponents. Scarf (1971) proved the non-emptiness of the $\alpha$-core when the players are endowed with continuous and quasi-concave utility functions and the sets of strategies are finite-dimensional. Kajii (1992) extended Scarf's result to the setting of games with continuous and nonordered preferences: each player is characterized by a mapping defined on the set of strategy profiles, and it is assumed that all such mappings are open-graph and convexvalued.

Vincenzo Scalzo

scalzo@unina.it

Achille Basile

basile@unina.it

1 Department of Economics and Statistics (DISES), University of Naples Federico II, Via Cinthia 21, 80126 Naples, Italy 
In the light of some recent advances on the existence of Nash equilibria in discontinuous games, new conditions for the non-emptiness of the $\alpha$-core in games with discontinuous payoff functions have been provided by Uyanik (2015). In the mentioned paper, the author introduces a coalitional version of the continuous-security by Barelli and Meneghel (2013), and other conditions so that the non-emptiness of the $\alpha$-core is obtained through the non-emptiness of the core of a suitable $N T U$-game. Here, Scarf's Theorem (Scarf 1967) plays a crucial role.

In the present paper, we give necessary and sufficient conditions for the nonemptiness of the $\alpha$-core in games with discontinuous and non-ordered preferences. In doing this, we do not use Scarf's Theorem (Scarf 1967), but we adapt to our setting the approach to the Nash equilibrium existence problem considered by Scalzo (2019a). More precisely, given a game with non-ordered preferences, we define a real-valued function so that the solutions to the corresponding Ky Fan minimax inequality (Fan 1972) coincide with the $\alpha$-core elements of the game. Hence, we apply the general results on the existence of solutions to the Ky Fan minimax inequality given by Scalzo (2013, 2019a) and identify sufficient (and necessary) conditions on the game for the non-emptiness of the $\alpha$-core. The conditions we obtain are adaptations to our setting of those given by Scalzo (2019a, b) for the existence of Nash equilibria in discontinuous games.

The investigation on the non-emptiness of the $\alpha$-core in games with discontinuous and non-ordered preferences can be motivated, for example, by the analysis of situations like the following one. Consider a family of clubs $\left\{C_{i}: i \in N\right\}$, each of which being represented by an individual $i \in N$, called administrator of $C_{i}$. Suppose that $i \neq j$ implies $C_{i} \neq C_{j}$. Assume that clubs get benefits from a common asset and the gain they obtain depends on the investments of all clubs. So, every club ranks profile of investments $x=\left(x_{i}\right)_{i \in N}$ by means of a rule which aggregates the preferences of the members (for example, unanimity rule, majority rule, etc.). Even if each member of a club has a utility function on the set of profiles of investments, the aggregation rule may give rise to a non necessarily ordered preference (for instance, the majority rule induces a non transitive preference). Moreover, the aggregate preference of a club $C_{i}$ may not satisfy the continuity properties considered by the previous literature (open-graph preferences). This is the case, for example, if $i$ is interpreted as the administrator of a condominium $C_{i}$. By the role, she/he is naturally led to identify amounts even when they are different but differ by less than a threshold level $\lambda>0$. That is: $x$ and $y$ are indifferent for $C_{i}$ if $\left|\sum_{j \in N} x_{j}-\sum_{j \in N} y_{j}\right|<\lambda$; and $C_{i}$ prefers $y$ on $x$ if $\sum_{j \in N} y_{j} \geq \sum_{j \in N} x_{j}+\lambda$ (see also Campbell and Walker 1990). This preference cannot be represented by utility functions and the mapping $P_{i}$ defined by $P_{i}(x)=\left\{y: C_{i}\right.$ prefers $y$ on $\left.x\right\}$ is not open-graph. ${ }^{1}$ Now, remaining in the condominium interpretation, it is plausible that all $C_{i}$ are located in a common district, reason why cooperation among administrators is beneficial. Consequently, the $\alpha$-core solution concept plays obviously a role, whereas the results from the previous literature do not apply.

\footnotetext{
${ }^{1}$ One can easily see that the symmetric part of the preference is not transitive. Moreover, $\bar{x} \in P_{i}(x)$ for $\bar{x}$ given by $\bar{x}_{j}=x_{j}+\frac{\lambda}{|N|}$ for each $j \in N$. Now, it is clear that there are profiles of investments $x^{\prime}$ indifferent to $\bar{x}$ in every open neighborhood of $x$. This implies that the graph of $P_{i}$ is not open.
} 
The paper is organized as follows. Section 2 recalls definitions and results on the Ky Fan minimax inequality. The setting of the paper is introduced in Sect. 3, where the previous literature on the non-emptiness of the $\alpha$-core is also recalled. Our main results, as well as the comparison with the previous ones, are given in Sect. 4. Section 5 concludes the paper.

\section{Ky Fan minimax inequality background}

Let $X$ be a non-empty subset of a topological vector space and $\Theta$ be a real-valued function defined on $X \times X$. The following problem

$$
(\mathrm{KF})\left\{\begin{aligned}
\text { find } x^{*} \in X & \text { such that } \\
\Theta\left(x, x^{*}\right) \leq 0 & \forall x \in X
\end{aligned}\right.
$$

is called Ky Fan minimax inequality corresponding to the function $\Theta$ (Ky Fan 1972). An element $x^{*}$, if any exists satisfying condition (KF), is a solution to the inequality; let $S_{\Theta}$ denote the set of solutions. Below, we present some properties which play a role in the existence of solutions to $(\mathrm{KF})$.

Definition 2.1 (Scalzo 2019a) The function $\Theta$ is said to be slightly diagonally transfer continuous if $\Theta(x, z)>0$ implies that there exists an open neighborhood $O_{z}$ of $z$ and $x^{\prime} \in X$ so that $\Theta\left(x^{\prime}, z^{\prime}\right)>0$ for all $z^{\prime} \in O_{z} \backslash S_{\Theta}$.

Definition 2.2 The function $\Theta$ is said to be generalized lower transfer continuous if $\Theta(x, z)>0$ implies that there exists an open neighborhood $O_{z}$ of $z$ and an upper semicontinuous mapping $\xi: O_{z} \rightrightarrows X$, with non-empty, convex and compact values, so that $\Theta\left(x^{\prime}, z^{\prime}\right)>0$ for all $z^{\prime} \in O_{z} \backslash S_{\Theta}$ and all $x^{\prime} \in \xi\left(z^{\prime}\right)$.

The property in Definition 2.1 generalizes the diagonal transfer continuity by Baye et al. (1993) because it allows a non-solution to (KF) to be approached by nets of solutions. In the same spirit, Definition 2.2 presents a generalization of the generalized 0-quasi-transfer continuity given in Definition 2 by Scalzo (2013). It is clear that every slightly diagonally transfer continuous function is generalized lower transfer continuous, but the converse does not hold: see the example below.

Example 2.1 Let $u$ be the real-valued function defined on $X=[0,1] \times[0,1]$ as follows:

$$
u\left(x_{1}, x_{2}\right)=\left\{\begin{aligned}
x_{1} & \text { if } x_{1}=x_{2}>0 \\
-1 & \text { if } x_{1}=x_{2}=0 \\
0 & \text { otherwise. }
\end{aligned}\right.
$$

Define $\Theta(x, z)=u\left(x_{1}, z_{2}\right)-u(z)$ for all $x$ and $z$ belonging to $X$. We have $S_{\Theta}=$ $\left.\left.\left\{x: x_{1}=x_{2}>0\right\} \cup(] 0,1\right] \times\{0\}\right)$. Note that the non solution $(0,0)$ is the limit (in the usual topology) of sequences of solutions to (KF). The function $\Theta$ is generalized lower transfer continuous. In fact, let $\Theta(x, z)>0$ for some $x$ and $z$. If $z \neq(0,0)$, the inequality is possible only if $z_{1} \neq z_{2}$ and $x_{1}=z_{2}>0$. It is easy to see that 
the generalized lower transfer continuity is satisfied for an open neighborhood $O_{z}$ of $z$ where $z_{1}^{\prime} \neq z_{2}^{\prime}$ for all $z^{\prime} \in O_{z}$ and $\xi\left(z^{\prime}\right)=\left\{z_{2}^{\prime}\right\} \times[0,1]$ for each $z^{\prime} \in O_{z}$. If $z=(0,0)$ and $O_{z}$ is an open neighborhood of $z$, since every $z^{\prime} \in O_{z} \backslash S_{\Theta}$ is such that $z_{1}^{\prime} \neq z_{2}^{\prime}$, by using the mapping $\xi$ defined above, we recognize that $\Theta$ is generalized lower transfer continuous (since $(0,0)$ is approached by sequences of solutions to the Ky Fan minimax inequality, the function $\Theta$ is not generalized 0-quasi-transfer continuous). Finally, in order to have $\Theta\left(x^{\prime}, z^{\prime}\right)>0$ for all $z^{\prime} \in O_{z} \backslash S_{\Theta}$, it is clear that $x^{\prime}$ must change as $z^{\prime}$ does. So, $\Theta$ is not slightly diagonally transfer continuous.

Definition 2.3 (Baye et al. 1993) The function $\Theta$ is said to be diagonally transfer quasi-concave if, for any $\left\{x^{1}, \ldots, x^{k}\right\} \subset X$ there exists $\left\{z^{1}, \ldots, z^{k}\right\} \subset X$, where $x^{h} \mapsto z^{h}$, such that, for each $z \in \operatorname{co}\left\{z^{1}, \ldots, z^{k}\right\}$ and $z=\sum_{j=1}^{l} \lambda_{j} z^{h_{j}}$ with $\lambda_{j}>0$ for $j=1, \ldots, l$, one has $\Theta(x, z) \leq 0$ for some $x \in\left\{x^{h_{1}}, \ldots, x^{h_{l}}\right\}$.

Definition 2.4 (Zhou and Chen 1988) The function $\Theta$ is said to be 0-diagonally quasi-concave if, for any $\left\{x^{1}, \ldots, x^{k}\right\} \subset X$ and any $z \in \operatorname{co}\left\{x^{1}, \ldots, x^{k}\right\}$ with $z=\sum_{j=1}^{l} \lambda_{j} x^{h_{j}}$ and $\lambda_{j}>0$ for $j=1, \ldots, l$, there exists $x \in\left\{x^{h_{1}}, \ldots, x^{h_{l}}\right\}$ so that $\Theta(x, z) \leq 0$.

Obviously, every 0-diagonally quasi-concave function is diagonally transfer quasiconcave, but the converse does not hold.

We have the following results on the existence of solutions to (KF).

Proposition 2.1 (Scalzo 2019a) Assume that X is a convex and compact subset of a Hausdorff topological vector space and $\Theta$ be slightly diagonally transfer continuous. Then, $S_{\Theta}$ is non-empty if and only if $\Theta$ is diagonally transfer quasi-concave.

Proposition 2.2 Assume that $X$ is a convex and compact subset of a locally convex Hausdorff topological vector space. If $\Theta$ is generalized lower transfer continuous and 0-diagonally quasi-concave, then $S_{\Theta}$ is non-empty.

Proof By contradiction, assume $S_{\Theta}=\emptyset$. Because of the generalized lower transfer continuity and $S_{\Theta}=\varnothing$, one has that $\Theta$ is generalized 0-quasi-transfer continuous. So, Proposition 2 by Scalzo (2013) applies and we get $S_{\Theta} \neq \emptyset$, which is a contradiction. $^{2}$

Remark 2.1 In Sect. 4, we provide $\alpha$-core non-emptiness results by using the Ky Fan minimax inequality. So, in the light of Proposition 4.2 and Remark 4.2, one can deduce that Proposition 2.2 cannot be obtained from Proposition 2.1, and viceversa.

\section{Setting and preliminaries}

Let $N$ be a finite set of players and, for each $i \in N$, let $X_{i}$ be a non-empty set. Each player $i$ is endowed with a mapping $P_{i}: X=\prod_{j \in N} X_{j} \rightrightarrows X$. Given $x \in X, P_{i}(x)$

\footnotetext{
2 Proposition 2 (Scalzo 2013). Assume that $X$ is a convex and compact subset of a locally convex Hausdorff topological vector space. If $\Theta$ is generalized 0 -quasi-transfer continuous and 0 -diagonally quasi-concave, then $S_{\Theta}$ is non-empty and compact.
} 
represents the set of all strategy profiles that player $i$ ranks better than $x$. For instance, if player $i$ compares strategy profiles by means of a binary relation and $\succ_{i}$ denotes its asymmetric part, we have $P_{i}(x)=\left\{z \in X: z \succ_{i} x\right\}$. In the same way, if $u_{i}$ is a utility function of player $i$ on $X, P_{i}(x)=\left\{z \in X: u_{i}(z)>u_{i}(x)\right\}$. However we point out that the mapping $P_{i}$ does not necessarily come from an ordered preference. The list $G=\left\langle X_{i}, P_{i}\right\rangle_{i \in N}$ of data is named strategic form game with non-ordered preferences (game, briefly).

Given a game $G$, we call coalition every non-empty subset of players; $\mathcal{N}$ denotes the set of coalitions of $G$. For each $S \in \mathcal{N}$, let $X_{S}=\prod_{j \in S} X_{j}$ and $X_{-S}=\prod_{j \notin S} X_{j}$; a strategy profile $x \in X$ will be also denoted by $x=\left(x_{S}, x_{-S}\right)$.

In this paper, we are interested in the cooperative solution concept for games proposed by Aumann (1961) and recalled in the following definition:

Definition 3.1 Let $G$ be a game. The strategy profile $z \in X$ is said to be $\alpha$-blocked if there exists a coalition $S$ and $x_{S} \in X_{S}$ such that $\left\{x_{S}\right\} \times X_{-S} \subseteq \bigcap_{i \in S} P_{i}(z)$. The set of all non $\alpha$-blocked strategy profiles is called $\alpha$-core of $G$ and is denoted by ${ }^{\alpha} \mathcal{C}(G)$.

The first result on the non-emptiness of the $\alpha$-core was given by Scarf (1971) for games whose players are endowed with continuous, quasi-concave utilities, and the strategy sets are finite-dimensional. Later, Kajii (1992) extended the result by Scarf (1971) to the games where the players' preferences are non-ordered and the sets of strategies are included in normed vector spaces. In this result, the mappings of players are assumed to be open graph and convex-valued. More recently, in the framework of finite-dimensional spaces of strategies, Uyanik (2015) obtained the non-emptiness of the $\alpha$-core in normal form games with quasi-concave payoff functions: inspired by Barelli and Meneghel (2013), the author considered a condition on payoffs which allows discontinuities.

Let us note that Scarf's Theorem (Scarf 1967) on the non-emptiness of the core of NTU-games plays a significant role in all the above mentioned papers. The approach of the present paper is different and we do not need Scarf's Theorem for our existence result.

\section{Non-emptiness of the alpha-core}

In this section, we give sufficient and necessary conditions for the non-emptiness of the $\alpha$-core in the framework of discontinuous games. We deal with games with nonordered preferences. To do this, we consider a suitable real-valued function $\Theta$ such that the solution set to the Ky Fan minimax inequality corresponding to $\Theta$ coincides with the $\alpha$-core.

Let $G=\left\langle X_{i}, P_{i}\right\rangle_{i \in N}$ be a game with non-ordered preferences. Define the function $\Theta$ on $X \times X$ as follows:

$$
\Theta(x, z)=\sum_{S \in \mathcal{N}} \min _{y_{-S} \in X_{-S}} \min _{i \in S} \widehat{P}_{i}\left(\left(x_{S}, y_{-S}\right), z\right),
$$

where $\widehat{P}_{i}\left(\left(x_{S}, y_{-S}\right), z\right)=1$ if $\left(x_{S}, y_{-S}\right) \in P_{i}(z)$ and $\widehat{P}_{i}\left(\left(x_{S}, y_{-S}\right), z\right)=0$ otherwise. 
We have: $x^{*} \in{ }^{\alpha} \mathcal{C}(G)$ if and only if $\Theta\left(x, x^{*}\right)=0$ for all $x \in X$. Indeed, if $\Theta\left(x, x^{*}\right)=0$ for all $x \in X$, we have $\min _{y_{-S} \in X_{-S}} \min _{i \in S} \widehat{P}_{i}\left(\left(x_{S}, y_{-S}\right), x^{*}\right)=0$ for each coalition $S$. This implies that, for every strategy profile $x$ and every coalition $S$, there exists $y_{-S} \in X_{-S}$ and $i \in S$ such that $\left(x_{S}, y_{-S}\right) \notin P_{i}\left(x^{*}\right)$. In other words: no coalition can use a joint deviation to $\alpha$-block $x^{*}$. So, $x^{*}$ is an $\alpha$-core element. Similarly, one gets that $x^{*}$ is a solution to the Ky Fan minimax inequality corresponding to the function $\Theta$ if $x^{*} \in{ }^{\alpha} \mathcal{C}(G)$.

Since $\Theta \geq 0$, the $\alpha$-core of $G$ coincides with the solution set to the Ky Fan minimax inequality corresponding to $\Theta$.

The following definitions introduce to our framework some deviation properties used in recent Nash equilibrium existence results (see Remark 4.1).

Definition 4.1 A game $G$ is said to satisfy the coalitional deviation property if, for all $z \notin{ }^{\alpha} \mathcal{C}(G)$, there exists an open neighborhood $O_{z}$ of $z$ and $x^{\prime} \in X$ such that, for any $z^{\prime} \in O_{z} \backslash{ }^{\alpha} \mathcal{C}(G)$, there exists a coalition $S$ for which $\left\{x_{S}^{\prime}\right\} \times X_{-S} \subseteq P_{i}\left(z^{\prime}\right)$ for every $i \in S$.

Definition 4.2 A game $G$ is said to satisfy the generalized coalitional deviation property if, for all $z \notin{ }^{\alpha} \mathcal{C}(G)$, there exists an open neighborhood $O_{z}$ of $z$ and an upper semicontinuous mapping $\xi_{z}: O_{z} \rightrightarrows X$, with non-empty, convex and compact values, such that, for each $z^{\prime} \in O_{z} \backslash{ }^{\alpha} \mathcal{C}(G)$ and each $x^{\prime} \in \xi_{z}\left(z^{\prime}\right)$, there exists a coalition $S$ for which $\left\{x_{S}^{\prime}\right\} \times X_{-S} \subseteq P_{i}\left(z^{\prime}\right)$ for every $i \in S$.

Remark 4.1 The definitions above are given in the spirit of the slight single deviation property and the generalized single deviation property introduced by Scalzo (2019a, b) in the framework of the Nash equilibrium existence problem, where new existence results have been obtained. The properties are generalizations of the single deviation property given by Nassah and Tian (2008) and Reny (2009). As it is showed in the following, the coalitional versions of the mentioned properties allow to obtain $\alpha$-core non-emptiness results in the setting of discontinuous games.

The class of games which satisfy the coalitional deviation property strictly includes the class of games where the mappings $P_{i}$ are open graph, as the following Proposition 4.1 and Example 4.1 show.

Proposition 4.1 Let $G=\left\langle X_{i}, P_{i}\right\rangle_{i \in N}$ be a game. If $P_{i}$ is open graph for all $i \in N$, then $G$ satisfies the coalitional deviation property.

Proof Suppose that $z \notin{ }^{\alpha} \mathcal{C}(G)$. So, for some coalition $S$ and $x_{S} \in X_{S}$, we get $\left\{x_{S}\right\} \times X_{-S} \subseteq P_{i}(z)$ for all $i \in S$, that is: $\{z\} \times\left(\left\{x_{S}\right\} \times X_{-S}\right) \subseteq G r\left(P_{i}\right){ }^{3}$ For every $i \in S$, since $P_{i}$ is open graph, there exists an open neighborhood $O_{z}^{i}$ of $z$ and an open set $A^{i} \subseteq X$, which includes $\left\{x_{S}\right\} \times X_{-S}$, such that $O_{z}^{i} \times A^{i} \subseteq \operatorname{Gr}\left(P_{i}\right)$. This implies that $\left\{z^{\prime}\right\} \times\left(\left\{x_{S}\right\} \times X_{-S}\right) \subseteq G r\left(P_{i}\right)$ for all $z^{\prime} \in O_{z}^{i}$. Now, set $O_{z}=\bigcap_{i \in S} O_{z}^{i}$ and let $x^{\prime}$ be a point in $X$ such that $x_{S}^{\prime}=x_{S}$. We get $\left\{x_{S}^{\prime}\right\} \times X_{-S} \subseteq P_{i}\left(z^{\prime}\right)$ for all $i \in S$ and all $z^{\prime} \in O_{z}$, which proves that the coalition deviation property is satisfied (in the present situation, the coalition which $\alpha$-blocks $z^{\prime} \in O_{z}$ does not depend on $z^{\prime}$ ).

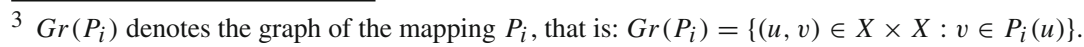


Example 4.1 Consider the normal form game with discontinuous and non quasiconcave payoff functions $G=\left\langle X_{i}, u_{i}\right\rangle_{i=1,2}$ (borrowed from Example 2 by Scalzo 2019a) such that: $X_{1}=X_{2}=[0,1]$ and $u(x)=\left(u_{1}(x), u_{2}(x)\right)$ is defined as below

$$
u(x)= \begin{cases}(0,1) & \text { if } x_{1}<x_{2} \text { and } x \notin B \\ (1,0) & \text { if } x_{1}>x_{2} \text { and } x \notin B \\ (0,0) & \text { if } x_{1}=x_{2}=0 \\ (1,1) & \text { if } x \in B\end{cases}
$$

where $\left.\left.\left.\left.B=\left\{x: x_{1}=x_{2}>0\right\} \cup(\{0\} \times] 0,1\right]\right) \cup(] 0,1\right] \times\{0\}\right)$. Define $P_{i}(x)=\{z$ : $\left.u_{i}(z)>u_{i}(x)\right\}$. It is easy to check that ${ }^{\alpha} \mathcal{C}(G)=B$. Moreover, in order to see that the coalitional deviation property holds true, it is sufficient to set $x^{\prime}=(1,1)$ for every $z \notin{ }^{\alpha} \mathcal{C}(G)$. Finally, it is obvious that $P_{i}$ is not an open-graph mapping.

The following example proves that the property introduced in Definition 4.2 strictly generalizes that given in Definition 4.1

Example 4.2 Let $G=\left\langle X_{i}, P_{i}\right\rangle_{i=1,2}$ be the game where $X_{1}=X_{2}=[0,1]$ and

$$
P_{i}(x)=\left\{\begin{aligned}
\left\{x_{-i}\right\} \times[0,1] & \text { if } x_{i}>x_{-i} \\
\emptyset & \text { otherwise }
\end{aligned}\right.
$$

One can see that ${ }^{\alpha} \mathcal{C}(G)=\left\{x: x_{1}=x_{2}\right\}$. Now, if $z \notin{ }^{\alpha} \mathcal{C}(G)$, for some $i \in\{1,2\}$, we have $z_{i}>z_{-i}$. So, there exists an open neighborhood $O_{z}$ such that $z_{i}^{\prime}>z_{-i}^{\prime}$ for all $z^{\prime} \in O_{z}$ and, by setting $\xi_{z}\left(z^{\prime}\right)=\left\{z_{-i}^{\prime}\right\} \times[0,1]$ for all $z^{\prime} \in O_{z}$, one recognizes that the generalized coalitional deviation property holds true. Moreover, only player $i$ can $\alpha$-block every $z^{\prime} \in O_{z}$, and the strategy he uses depends on $z^{\prime}$. Hence, the game does not satisfy the coalitional deviation property.

The definitions below give coalitional versions of some quasi-concavity like properties employed to solve the Nash equilibrium existence problem in ordinal and normal form games. We refer to the transfer uniform quasi-concavity by Nassah and Tian (2016) and Scalzo (2019a) and the $\mathcal{C}$-condition by Scalzo (2019b). Given a subset $A$ of a vector space, with $\operatorname{sco} A$ we denote the set of all strict convex combinations of all elements of $A$.

Definition 4.3 A game $G$ is said to be coalitional transfer quasi-concave if, for each $\left\{x^{1}, \ldots, x^{k}\right\} \subset X$ there exists $\left\{z^{1}, \ldots, z^{k}\right\} \subset X$, where $x^{h} \mapsto z^{h}$, such that, for every $z \in \operatorname{sco}\left\{z^{h_{1}}, \ldots, z^{h_{l}}\right\}$, with $\left\{z^{h_{1}}, \ldots, z^{h_{l}}\right\} \subseteq\left\{z^{1}, \ldots, z^{k}\right\}$, for at least one $x \in$ $\left\{x^{h_{1}}, \ldots, x^{h_{l}}\right\}$ we have that no coalition can $\alpha$-block $z$ by using $x$, that is: for all $S \in \mathcal{N}$, there is $w_{-S} \in X_{-S}$ and $i \in S$ so that $\left(x_{S}, w_{-S}\right) \notin P_{i}(z)$.

Definition 4.4 A game $G$ is said to be coalitional quasi-concave if, for each $\left\{x^{1}, \ldots, x^{k}\right\} \subset X$ and each $z \in \operatorname{sco}\left\{x^{1}, \ldots, x^{k}\right\}$, there exists $x \in\left\{x^{1}, \ldots, x^{k}\right\}$ so that no coalition can $\alpha$-block $z$ by using $x$.

It is clear that coalitional quasi-concavity implies coalitional transfer quasi-concavity. The coalitional transfer quasi-concavity is a necessary condition for the non-emptiness 
of the $\alpha$-core. Indeed, if $x^{*} \in{ }^{\alpha} \mathcal{C}(G)$, to see that the property is satisfied, it is sufficient to set $\left\{z^{1}, \ldots, z^{k}\right\}=\left\{x^{*}\right\}$ for every subset of strategy profiles $\left\{x^{1}, \ldots, x^{k}\right\}$. However, the coalitional transfer quasi-concavity is not enough to guarantee the non-emptiness of the $\alpha$-core, as the following example shows.

Example 4.3 Consider the 2-player game with mappings $P_{1}$ and $P_{2}$ defined on $[0,1] \times$ $[0,1]$ as follows:

$$
P_{1}(x)=\left\{\begin{array}{rll}
] x_{1}, 1\right] \times[0,1] & \text { if } x_{1} \in[0,1[ & P_{2}(x) \equiv \emptyset . \\
] 0,1[\times[0,1] & \text { if } x_{1}=1 &
\end{array}\right.
$$

It is easy to see that ${ }^{\alpha} \mathcal{C}(G)=\emptyset$. Moreover, the game is coalitional quasi-concave.

It is interesting to note that, if one looks at the function $\Theta$ defined by means of (4.1), the properties introduced in Definitions 4.1, 4.2, 4.3 and 4.4 can be reformulate in terms of properties recalled in Sect. 2 for the $(\mathrm{KF})$ inequality. In particular, we have the following proposition, whose proof is obvious.

Proposition 4.2 Let $G$ be a game and let $\Theta$ be defined by (4.1). One has:

(i) G satisfies the coalitional deviation property if and only if $\Theta$ is slightly diagonally transfer continuous.

(ii) $G$ satisfies the generalized coalitional deviation property if and only if $\Theta$ is generalized lower transfer continuous.

(iii) $G$ is coalitional transfer quasi-concave if and only if $\Theta$ is diagonally transfer quasi-concave.

(iv) $G$ is coalitional quasi-concave if and only if $\Theta$ is 0-diagonally quasi-concave.

Now, from Proposition 4.2, in the light of Propositions 2.1 and 2.2 , we obtain the following results on the non-emptiness of the $\alpha$-core in games with non-ordered preferences.

Theorem 4.1 Assume that $X_{i}$ is a convex and compact subset of a Hausdorff topological vector space, for each $i \in N$. Let $G=\left\langle X_{i}, P_{i}\right\rangle_{i \in N}$ be a game that satisfies the coalitional deviation property. Then, ${ }^{\alpha} \mathcal{C}(G)$ is non-empty if and only if $G$ is coalitional transfer quasi-concave.

Theorem 4.2 Assume that $X_{i}$ is a convex and compact subset of a locally convex Hausdorff topological vector space for each $i \in N$. Let $G=\left\langle X_{i}, P_{i}\right\rangle_{i \in N}$ be a game that satisfies the generalized coalitional deviation property and is coalitional quasiconcave. Then ${ }^{\alpha} \mathcal{C}(G)$ is non-empty.

Remark 4.2 Theorem 4.1 presents a class of discontinuous games (games satisfying the coalitional deviation property) where the non-emptiness of the $\alpha$-core is characterized by means of a quasi-concavity like property (the coalitional transfer quasi-concavity). In Theorem 4.2, the coalitional deviation property is replaced with a more general condition; at the same time, the coalitional transfer quasi-concavity is strengthened with the coalitional quasi-concavity. However, Theorems 4.1 and 4.2 are each other independent. 
For instance, the game presented in Example 4.2, which satisfies the generalized coalitional deviation property but not the coalitional deviation property, is coalitional quasi-concave. To prove this, we proceed by contradiction and assume that there exists $z \in \operatorname{sco}\left\{x^{1}, \ldots, x^{k}\right\}$ such that: for each $x \in\left\{x^{1}, \ldots, x^{k}\right\}$ there is a coalition $S$ so that $\left\{x_{S}\right\} \times X_{-S} \subseteq P_{i}(z)$ for all $i \in S$. If $z_{1}=z_{2}$, we have that $z \in{ }^{\alpha} \mathcal{C}(G)$, and this is impossible. So, we have that $z_{1} \neq z_{2}$. Suppose $z_{1}>z_{2}$. Only coalition $\{1\}$ can $\alpha$ blocks $z$, and we obtain $\left\{x_{1}\right\} \times[0,1] \subseteq P_{1}(z)=\left\{z_{2}\right\} \times[0,1]$ for all $x \in\left\{x^{1}, \ldots, x^{k}\right\}$, which implies $x_{1}^{h}=z_{2}$ with $h=1, \ldots, k$. Since $z \in \operatorname{sco}\left\{x^{1}, \ldots, x^{k}\right\}$, we get the contradiction $z_{1}=z_{2}$. Similarly if $z_{1}<z_{2}$, and the coalitional quasi-concavity holds true. So, in this example, Theorem 4.2 applies but Theorem 4.1 not.

On the other hand, Theorem 4.1 gives a characterization of the non-emptiness of the $\alpha$-core, while Theorem 4.2 provides only sufficient conditions.

\subsection{Comparison with the previous literature}

Examples 4.1 and 4.2 provide games where our results apply but not those by Scarf (1971) and Kajii (1992): the payoff functions in the first example are not continuous and the mappings from the second one are not open-graph.

Concerning the non-emptiness of the $\alpha$-core in normal form games with discontinuous payoff functions, Theorem 1 by Uyanik (2015) assumes finite-dimensional spaces of strategies, quasi-concave payoffs and a condition called coalitionally $C$-security. This condition require that, if $z \notin{ }^{\alpha} \mathcal{C}(G)$, for each coalition $S$ there exist a numerical vector $\left(v_{i}\right)_{i \in S}, x_{S} \in X_{S}$ and an open neighborhood $O_{z}$ of $z$ such that:

(a) $u_{i}\left(x_{S}, w_{-S}\right) \geq v_{i}$ for all $w_{-S} \in X_{S}$ and all $i \in S$, and

(b) for each $z^{\prime} \in O_{z}$ there is a coalition $S$ so that $u_{i}\left(z^{\prime}\right)<v_{i}$ for all $i \in S$.

It is easy to see that the coalitionally $C$-security fails on the game by Example 4.1 because of the point $z=(0,0)$.

In the setting of games with discontinuous non-ordered preferences, Martins-daRocha and Yannelis (2011) introduced the following condition, called $\alpha$-continuity: the mapping $P_{S}: X \rightrightarrows X_{S}$, defined for each coalition $S$ by

$$
P_{S}(x)=\left\{z_{S} \in X_{S}:\left\{z_{S}\right\} \times X_{-S} \subseteq \bigcap_{i \in S} P_{i}(x)\right\} \forall x \in X,
$$

has open lower sections. They obtained the non-emptiness of the $\alpha$-core in games satisfying the $\alpha$-continuity and such that $x \notin \operatorname{co} P_{i}(x)$ for all $x \in X$ and all $i \in N$. This property can be characterized as follows:

Proposition 4.3 Let $G=\left\langle X_{i}, P_{i}\right\rangle_{i \in N}$ be a game. Then, $\alpha$-continuity holds true if and only if the following property is satisfied: a) for each $z \notin^{\alpha} \mathcal{C}(G)$, there exist an open neighborhood $O_{z}$ of $z$, a coalition $S$, and $x_{S}^{\prime} \in X_{S}$ such that $\left\{x_{S}^{\prime}\right\} \times X_{-S} \subseteq \bigcap_{i \in S} P_{i}\left(z^{\prime}\right)$ for all $z^{\prime} \in O_{z}$.

Proof Suppose that $\alpha$-continuity holds and $z \notin{ }^{\alpha} \mathcal{C}(G)$. So, at least one coalition $S$ $\alpha$-blocks $z$ by using some $x_{S} \in X_{S}$, that is: $\left\{x_{S}\right\} \times X_{-S} \subseteq \bigcap_{i \in S} P_{i}(z)$. Because 
of $\alpha$-continuity, we have $O_{z} \subseteq P_{S}^{-1}\left(x_{S}\right)$ for some open neighborhood $O_{z}$ of $z$. Set $x_{S}^{\prime}=x_{S}$, we get that the property $a$ ) is satisfied. Similarly one gets the viceversa.

Proposition 4.3 shows that the class of $\alpha$-continuous games is included in the class of games which satisfy the coalitional deviation property. Moreover, the inclusion is strict because the $\alpha$-continuity fails on the game introduced in Example 4.1; in fact, one has $P_{\{1,2\}}^{-1}(1,1)=\{(0,0)\}$.

\section{Conclusions}

This paper has provided new conditions which guarantee the non-emptiness of the $\alpha$-core in the setting of games with discontinuous and non-ordered preferences and infinite-dimensional spaces of strategies. In particular, we have introduced sufficient and necessary conditions for the existence of $\alpha$-core elements. Inspired by a recent literature on the existence of Nash equilibria in games with non-ordered preferences, our results have been obtained by means of the Ky Fan minimax inequality. More precisely, given a game $G$ with non-ordered preferences, we have defined a realvalued functions $\Theta$ such that a strategy profile $x^{*}$ belongs to the $\alpha$-core of $G$ if and only if $\Theta\left(x, x^{*}\right) \leq 0$ for all strategy profiles $x$. We have obtained two results. In the first one, we have proved that the $\alpha$-core of a game satisfying the coalitional deviation property is non-empty if and only if the game is coalitional transfer quasi-concave. In the second one, we have considered a condition more general than the coalitional deviation property and we have obtained the non-emptiness of the $\alpha$-core by using a strengthening of the coalitional transfer quasi-concavity.

Funding Open access funding provided by Università degli Studi di Napoli Federico II within the CRUICARE Agreement.

Open Access This article is licensed under a Creative Commons Attribution 4.0 International License, which permits use, sharing, adaptation, distribution and reproduction in any medium or format, as long as you give appropriate credit to the original author(s) and the source, provide a link to the Creative Commons licence, and indicate if changes were made. The images or other third party material in this article are included in the article's Creative Commons licence, unless indicated otherwise in a credit line to the material. If material is not included in the article's Creative Commons licence and your intended use is not permitted by statutory regulation or exceeds the permitted use, you will need to obtain permission directly from the copyright holder. To view a copy of this licence, visit http://creativecommons.org/licenses/by/4.0/.

\section{References}

Aumann RJ (1961) The core of a cooperative game without side payments. Trans Am Math Soc 98:539-552

Barelli P, Meneghel I (2013) A note on the equilibrium existence problem in discontinuous games. Econometrica 81:813-824

Baye MR, Tian G, Zhou J (1993) Characterizations of the existence of equilibria in games with discontinuous and non-quasiconcave payoffs. Rev Econ Stud 60:935-948

Campbell DE, Walker M (1990) Maximal elements of weakly continuous relations. J Econ Theory Vol 50(2):459-464

Fan K (1972) A minimax inequality and applications. In: Shisha O (ed) Inequalities, vol 3. Academic Press, New York 
Kajii A (1992) A generalization of Scarf's theorem: an $\alpha$-core existence theorem without transitivity or completeness. J Econ Theory 56:194-205

Martins-da-Rocha V.F, Yannelis N (2011) Nonemptiness of the alpha core. Working paper. Manchester School of Social Sciences, University of Manchester

Nassah R, Tian G (2008) The existence of equilibria in discontinuous games. IESEG School of Management, Mimeo

Nassah R, Tian G (2016) On the existence of Nash equilibrium in discontinuous games. Econ Theory 61(3):515-540

Reny PJ (2009) Further results on the existence of nash equilibria in discontinuous games. University of Chicago, Mimeo

Scalzo V (2013) Essential equilibria of discontinuous games. Econ Theory 54(1):27-44

Scalzo V (2019a) Equilibrium existence in games: slight single deviation property and Ky Fan minimax inequality. J Math Econ 82:197-201

Scalzo V (2019b) Continuity properties of the Nash equilibrium correspondence in a discontinuous setting. J Math Anal Appl 473:1270-1279

Scarf HE (1967) The core of an N person game. Econometrica 35:50-69

Scarf HE (1971) On the existence of a cooperative solution for a general class of n-person games. J Econ Theory 3:169-181

Uyanik M (2015) On the nonemptiness of the $\alpha$-core of discontinuous games: transferable and nontransferable utilities. J Econ Theory 158:213-231

Zhou J, Chen G (1988) Diagonal convexity conditions for problems in convex analysis and quasi-variational inequalities. J Math Anal Appl 132:213-225

Publisher's Note Springer Nature remains neutral with regard to jurisdictional claims in published maps and institutional affiliations. 